\title{
A Reduced Order Modeling approach for optimal allocation of Distributed Generation in power distribution systems
}

\author{
Raquel García-Blanco \\ Pedro Díez \\ (of Affiliation): LaCàN \\ Universidad Politécnica de Cataluña \\ Barcelona, Spain \\ raquel.garcia.blanco@upc.edu
}

\author{
Domenico Borzacchiello \\ Francisco Chinesta \\ (of Affiliation): GeM, UMR 6183 \\ École Centrale de Nantes \\ Nantes, France
}

\begin{abstract}
This paper presents an "offline-online" strategy for optimal allocation and sizing of Distributed Generation. In traditional optimization approaches, each function evaluation requires the solution of a power flow problem, which makes global optimality a computationally challenging goal. In the proposed strategy the power flow solver is invoked only once and a parametric solution is constructed with a monolithic solver. Despite the fact that the parametrized power flow equations result in a high-dimensional problem, the proposed algorithm is specifically designed to circumvent the curse of dimensionality. This is achieved through the application of Model Reduction, in particular the Proper Generalized Decomposition combined with a nonlinear solver. Numerical examples are carried out for showing the validity of the proposed method.
\end{abstract}

Index Terms-Distributed power generation, Model Reduction, Proper Generalized Decomposition, Optimal DG allocation.

\section{INTRODUCTION}

The optimal allocation of a distributed generator (DG) is a key feature to ensure the introduction of distributed generation in electrical power systems to be successful.

This consideration explains the intense research activity of the last decades aimed to the identification of optimization techniques in power grid analysis. In particular, [1] defines the optimal distributed generation placement problem (ODGP) claiming that it provides the best locations and sizes of DGs to optimize electrical distribution network operation and planning. When ODGP is solved, the objective function can be single or multi-objective. On one hand, the main single-objective functions are: minimization of energy losses, minimization of system average interruption duration index (SAIDI), minimization of cost, etc. On the other hand, in [2], ODGP multi-objective formulations are classified as multiobjective function with weights, where the multi-objective formulation is transformed into a single-objective function using the weighted sum of individual objectives; goal multi-objective index, where the multi-objective formulation is transformed into a single-objective function using the goal programming method and multi-objective formulation considering more than one often contrasting objectives.

As also in this paper, several studies have been focused on single-objective optimization, which in most cases corresponds to the minimization of the total power loss of the system. An example can be found in [3] where an implementation method of Tabu Search to find how much distribution loss can be reduced if DGs are optimally allocated is discussed. Authors in [4] and [5] proposed a genetic algorithm and an analytical method in order to minimize the total real power losses in the system. In [6] an analytical expression to calculate the optimal size and an effective methodology to identify the corresponding optimum location for DG placement for minimizing the total power losses in primary distribution systems is also presented. More recently, new methodologies have been proposed based on the optimal allocation of DG units in the distribution system so as to minimize annual energy loss [7][9]. Most optimization techniques require repeated evaluation of the objective function, each demanding the solution of a different power flow problem in order to compute the associated losses. When the number of parameters to optimize is high and global optimization is sought, this approach can become costly from the computational viewpoint.

The aim of this work is to illustrate a new strategy for optimal placement and sizing of DGs in power distribution systems based on a "offline-online" approach that separates the solution of the associated parametric power flow problem and the optimization into distinct steps. The novelty of this methodology is the fact that diverse parameters of the power flow problem are now considered as variables rather than input quantities. This perspective leads us to solve the power flow problem in a high-dimensional parametric space where the position of the DG, its generated power and the power demanded by the loads are regarded as additional coordinates. We address to this problem as the Parametric Power Flow (PPF).

PPF naturally arises as a high dimensional problem. In order to reduce the complexity of this problem we rely on Reduced 
Order Modelling (ROM). The ROM approach has been already successfully applied to a number of applications in power engineering, namely: optimization, uncertainty quantification and real time control [10]-[15].

Although in this paper we only take into account a single objective function, the concepts that are presented can be easily extended to multi-objective optimization. After solving the PPF problem once and for all, any particular objective function can be defined and the optimization step can be performed efficiently since it just requires inexpensive function evaluations and no longer power flow solutions.

The layout of the paper is organized as follows: an overview of the Proper Generalized Decomposition (PGD) method is given in section II, while section III illustrates the Parametric Power Flow problem and the iterative scheme including PGD for solving it. In section IV, numerical examples are presented for a problem in two and eight dimensions. Finally, the conclusions are discussed in section $\mathrm{V}$.

\section{PROPER GENERALIZED DECOMPOSITION}

The drawback of high dimensional problems is that the solution may become complicated due to the exponential increase of the degrees of freedom. Indeed, in $D$ dimensions if each parameter assumes $n$ possible states, the extensive

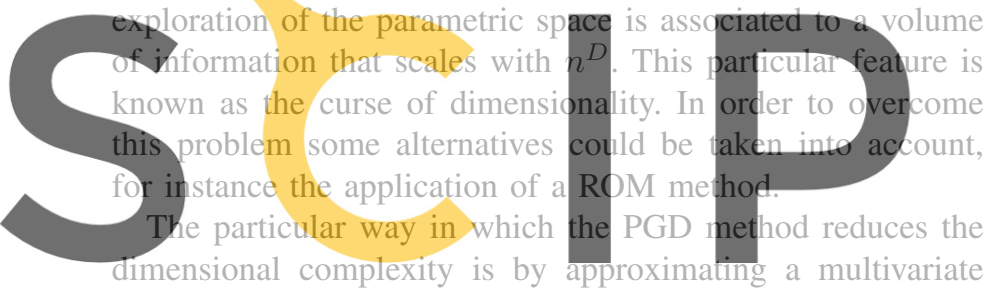
function by the sum of products of one-dimensional functions: Register for free at https//www.scipedia.com to

$$
V\left(s_{1}, s_{2}, \ldots, s_{n}\right) \approx \sum_{m}^{M} \nu_{1}^{m}\left(s_{1}\right) \nu_{2}^{m}\left(s_{2}\right) \ldots \mathcal{V}_{n}^{m}\left(s_{n}\right) .
$$

This form, called the separated variables representation, is computed using a greedy enrichment procedure, in which a single term per iteration is introduced in the summation. Each new term is determined using a fixed point algorithm in which each function is updated individually, using an alternating minimization approach.

The advantage of this approach is that the solution can be described by $n \cdot D \cdot M$ degrees of freedom instead of $n^{D}$ and that the algorithm has linear complexity with respect to $D$. Due to the important reduction in the complexity (from exponential to linear) the PGD approach is drastically alleviating the curse of dimensionality [16]-[19].

This strategy has been successfully applied to parametric problems in computational mechanics. For detailed information look at the references [20]-[22].

In general, the application of PGD to linear problems is straightforward, nevertheless the extension to nonlinear problems could be really arduous depending on the particular problem to be solved. In this work, the Z bus method [23] is applied to the power flow equations. This, can be seen as a particularization of the method of Alternating Search Directions (ASDM) [24]. This method is chosen because, unlike other classical methods such as Newton-Raphson, it is relatively easy to combine with PGD, since the nonlinear iteration is broken down into two linear steps that can be solved with PGD. For more details refer to [25].

\section{The PARAMetric Power Flow PROBlem}

The governing equations for the PPF problem and the corresponding separated variables forms are introduced in this section.

\section{A. Parametrized power flow equations}

When we consider the Parametric Power Flow problem, the voltage $V$, the power source $S$ and the current $I$ are no longer vectors of nodal values but vector valued functions of the problems parameters. Arranging all the parameters in a vector

$$
\xi=\left[s_{1}, s_{2}, \ldots, s_{n}\right]^{T}
$$

the parametrized Kirchhoff's current law writes as:

$$
\mathbf{Y} V(\xi)=I_{0}+I(\xi),
$$

where $\mathrm{Y}$ is the admittance matrix, $V$ is the vector of unknown

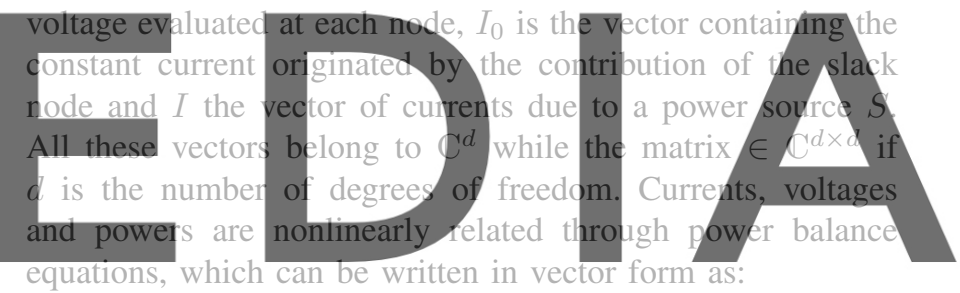

equations, which can be written in vector form as:

\section{download the suersioncmithout the watermark}

with $I^{*}$ being the complex conjugate of $I$ and the symbol

$\odot$ denoting the Hadamard product of vectors.

By incorporating (4) into (3), the following nonlinear system is obtained

$$
\mathbf{Y} V(\xi)=I_{0}+S(\xi)^{*} \oslash V(\xi)^{*},
$$

where the symbol $\oslash$ denotes the component-wise quotient between vectors.

\section{B. Separated variables representation}

In the remainder of this paper the following parameters are considered:

- The DG position $q$

- The nominal generated power $r$

- The time $t$, since the power flow analysis will be performed with time-varying loads over the period of a year.

Once the datum $S$ is expressed as a separated variables functions depending on $q, r$ and $t$

$$
S(r, t)=\sum_{h}^{H} \breve{Q}^{h} \breve{\mathcal{R}}^{h}(r) \breve{\mathcal{T}}^{h}(t),
$$


the objective of the PGD is to find the unknown voltage $V$ in a separated variables representation consisting of the sum of products of one-dimensional functions

$$
V(r, t) \approx V_{P G D}=\sum_{m}^{M} Q^{m} \mathcal{R}^{m}(r) \mathcal{T}^{m}(t),
$$

where $H, M$ are the number of terms in the summations, $\breve{\mathcal{R}}^{h}(r), \breve{\mathcal{T}}^{h}(t), \mathcal{R}^{m}(r)$ and $\mathcal{T}^{m}(t)$ are parametric modes. The modes express the functional dependency from the corresponding parameters $q, r, t$ and are recombined through the vectors coefficients $\breve{Q}^{h}$ and $Q^{m}$.

Once the solution $V_{P G D}$ is obtained, this can be used to generate a separated variables representation for the losses used for optimization:

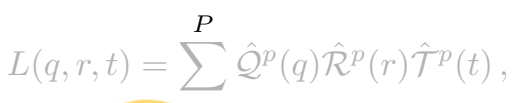

where $P$ is the number of terms in the sum, $\hat{\mathcal{Q}}^{p}(q), \hat{\mathcal{R}}^{p}(r)$ and $\hat{\mathcal{T}}^{p}(t)$ are parametric modes depending on the corresponding parameter $q, r$ and $t$.

C. Algorithm overview

Representing the variables in (5) in separated variables

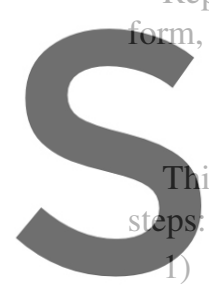

n, the $\mathrm{Z}$ bus method at itera

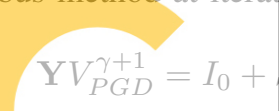

algorithm can be broken
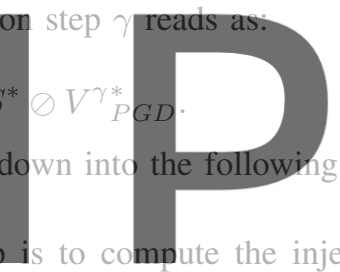

Given $V_{P G D}^{\gamma}$, the first step is to compute the injected
currents $I^{\gamma+1}$ in a separated variables form, evaluating Register for free at https//www.scipedia.com to $I^{\gamma+1}=S^{*} \oslash V_{P G D}^{\gamma *}$

(10)

Since the numerator $S$ and the denominator $V_{P G D}^{\gamma}$ are separated variables functions, the evaluation of the quotient is not straightforward, therefore PGD is applied to the associated linear problem

$$
V_{P G D}^{\gamma *} \odot I^{\gamma+1}=S^{*}
$$

in order to find a separated representation of $I^{\gamma+1}$. The PGD algorithm is stopped once the residual of equation (11) is smaller than a given tolerance parameter $\epsilon_{g}$. Each new mode in the summation is computed to a given precision $\epsilon_{f p}$.

2) The second step consists of solving the linear system

$$
V^{\gamma+1}=\boldsymbol{Y}^{-1}\left(I^{\gamma+1}+I_{0}\right)
$$

which does not present any additional difficulty because the matrix $\boldsymbol{Y}$ does not depend on the DG positioning or the power. For this reason, the factorization of the matrix can be stored and reused. Consequently, the modes for the voltage $V^{\gamma+1}$ can be straightforwardly computed by applying backward and forward substitution to the modes of $I^{\gamma+1}$.
The strategy presented is based on a particular iterative scheme and the necessity of an initial guess for the voltage comes up. This is taken as the voltage circulating in the grid under no loads or generation:

$$
V_{0}=\boldsymbol{Y}^{-1} I_{0}
$$

The nonlinear algorithm is terminated once the difference between the solution at two successive iterations is smaller than a tolerance parameter $\epsilon_{n}$.

\section{NUMERICAL APPLICATIONS}

In this section we show the proposed method for a test system. The network's diagram is shown in Fig. 1. The model, taken from [26], is a three-phase system with different topologies and load characteristics including a simplified representation of the high-voltage system. Some of the main characteristics of the substation transformer are given below:

- High-voltage rating: $230 \mathrm{kV}$

- Low-voltage rating: $4.16 \mathrm{kV}$

- Rated power of substation transformer: $10000 \mathrm{kVA}$.

The distributed generator is connected to the system through a step-up interconnection transformer allowing the switch from high-voltage to low-voltage. The authors of this work EDIA download the version without the watermark
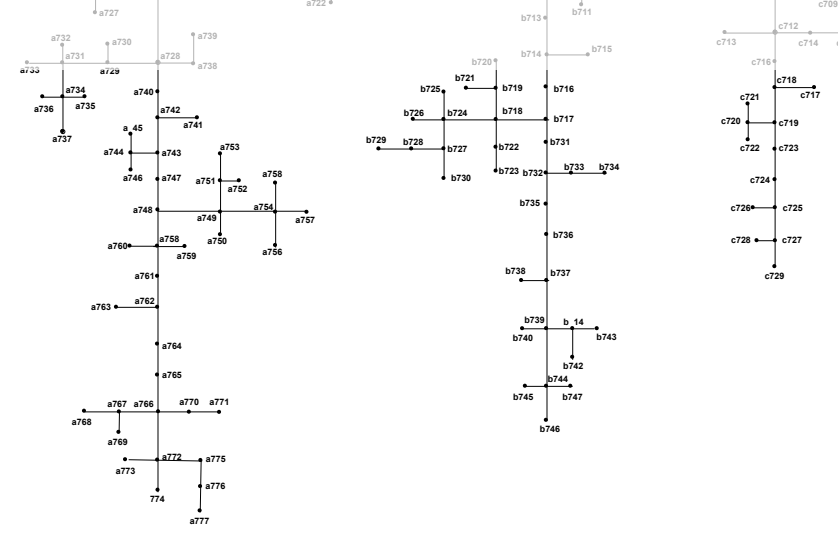

Fig. 1: Diagram of the test system

\section{A. Optimal positioning of a DG unit with a fixed loads}

We show as a first example the resolution of the Parametric Power Flow problem when two additional parameters being the position $q$ of the new DG and the generated active power $r$ are considered as variables. We seek the values of $q$ and $r$ that minimize the system losses. The problem consists in finding 
the separated form of the voltage solution at each node of the grid

$$
V(r) \approx \sum_{m}^{M} Q^{m} \mathcal{R}^{m}(r),
$$

$\forall q=1, \ldots, 82$ corresponding to the bus position in the two first branches in the network, and $\forall r$ in the set of possible values of active power that the DG can provide, that is in the partition of the interval $\left[0, r_{\max }\right]$ when the increment is $N_{r}=100$ (number of samples) and $r_{\max }=8 \cdot 10^{5} \mathrm{~W}$.

The approach "offline-online" mentioned in section III-C is followed. The tolerances are $\epsilon_{f p}=10^{-10}, \epsilon_{g}=10^{-8}$ and $\epsilon_{n}=10^{-6}$ and a solution could be found with 3 modes in just 6 nonlinear iterations as can be seen in Fig. 2, showing the convergence diagram of the method. In Fig. 3 the norm 2 of the 3 terms representing the solution are shown. These values quantify the contribution of each term of the summation in the separated representation of the solution, in this example the solution could be represented with sufficient precision just by the first 2 terms. It is worth mentioning that, if $N_{q}$ is the number of candidate positions where the DG can be positioned and $N_{d}$ is the number of degrees of freedom for the voltage nodes, the volume of information necessary to represent the whole parametric space is proportional to $N_{q} \times N_{r} \times N_{d} \approx$

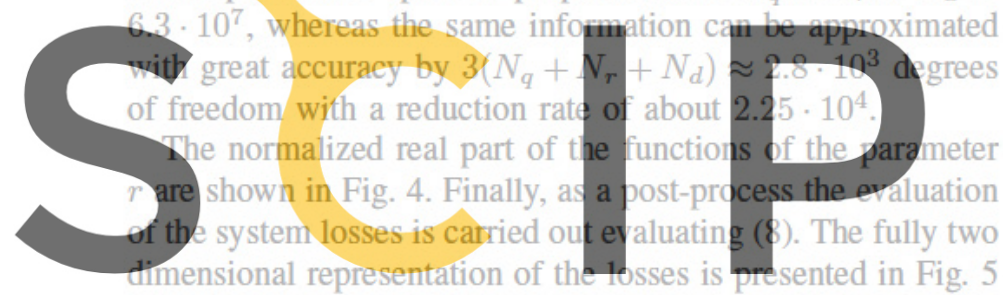
where we can observe that the minimum value of the losses are

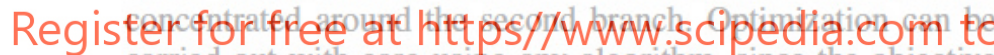
carried out with ease using any algorithm, since the objective function and its gradients are now explicitly available. In practice, the minimum ioss is $1.93 \cdot 10^{2} \mathrm{~W}$ when the $\mathrm{DG}$ is set at the position $l b 736$ with power $4.6 \cdot 10^{5} \mathrm{~W}$.

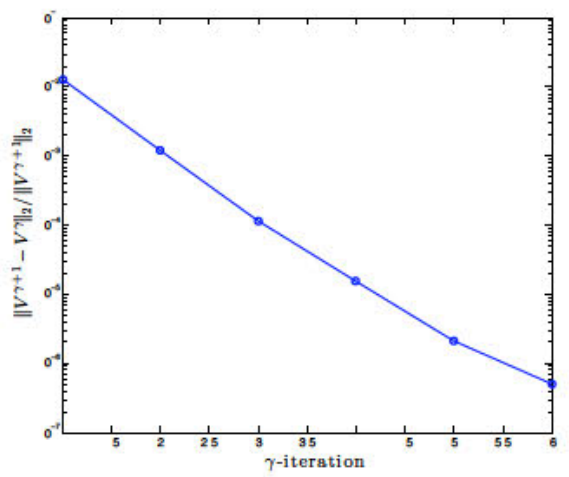

Fig. 2: Nonlinear PGD convergence diagram

\section{B. Optimal positioning of three DG units with time varying loads}

The goal in the last example is to minimize the power losses over a year when three DGs, one per branch, are set

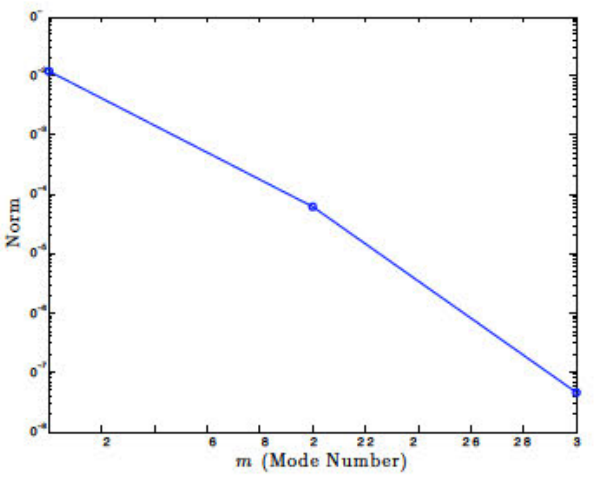

Fig. 3: Norm of the individual terms in the separated representation of $V$

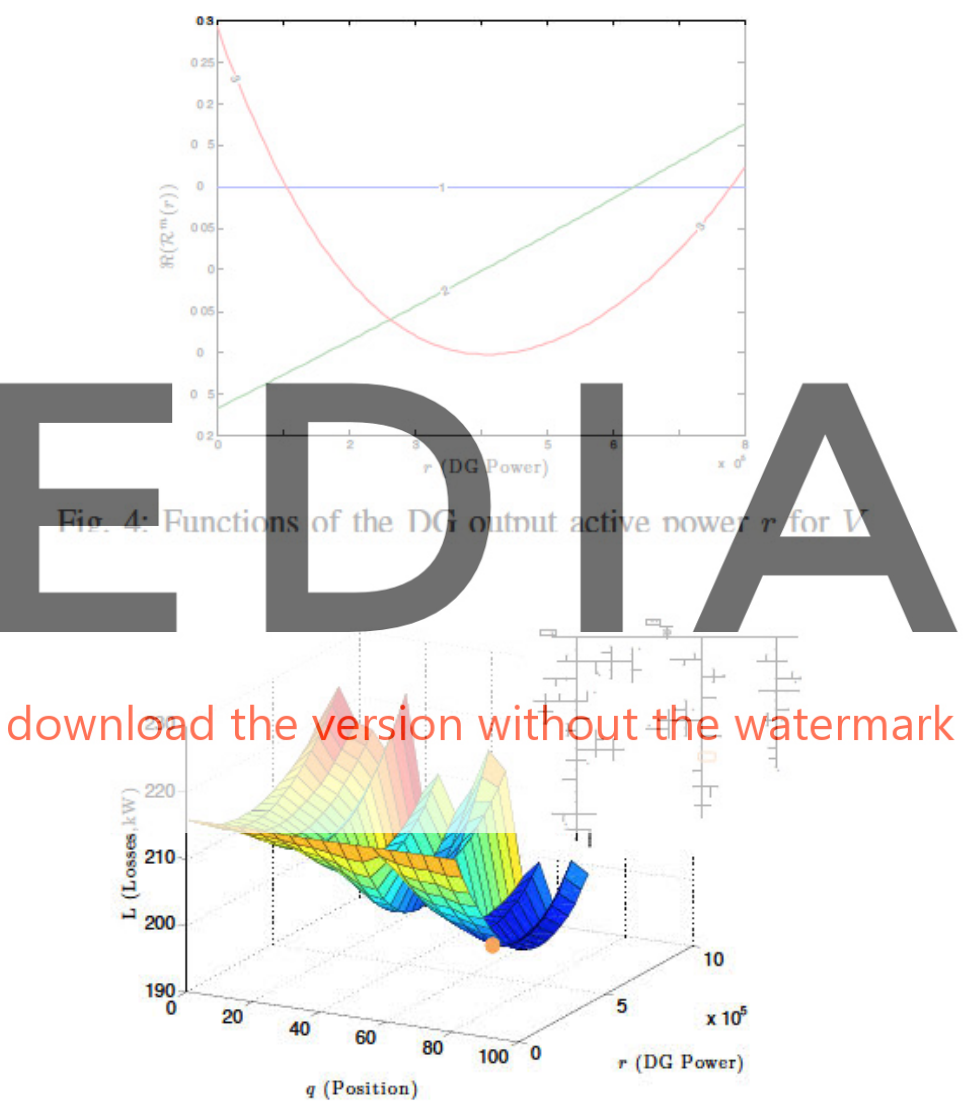

Fig. 5: Reconstructed System Losses

in the network. The parameters associated to the power flow problems are the position of the DGs $q_{1}, q_{2}$ and $q_{3}$, the active power of the DGs $r_{1}, r_{2}$ and $r_{3}$ and the time $t$. Considering also the nodal position where the voltage is computed, that is the physical coordinate of the system, the parametric problem is eight-dimensional. The representation of the solution does not always have to deal with one-dimension separated modes. Occasionally, considering functions that depend on more than one parameter is also efficient. In particular, although the problem is eight-dimensional the decomposition chosen is 4D3D-1D as can be seen in the representation of the input $S$, 


$$
\begin{array}{r}
S\left(q_{1}, q_{2}, q_{3}, r_{1}, r_{2}, r_{3}, t\right)=\mathcal{Q}^{1}\left(q_{1}\right) \mathcal{R}^{1}\left(r_{1}\right) \mathcal{T}^{1}(t) \\
+\mathcal{Q}^{2}\left(q_{2}\right) \mathcal{R}^{2}\left(r_{2}\right) \mathcal{T}^{2}(t)+\mathcal{Q}^{3}\left(q_{3}\right) \mathcal{R}^{3}\left(r_{3}\right) \mathcal{T}^{3}(t) \\
+\sum_{h=4}^{27} \breve{Q}^{h} \breve{\mathcal{R}}^{h}\left(r_{1}, r_{2}, r_{3}\right) \breve{\mathcal{T}}^{h}(t) .
\end{array}
$$

For the sake of simplicity, the parameters $q_{1}, q_{2}, q_{3}$ varying from the positions 1 to 19 at each branch. Similarly, due to the characteristics of the first two branches, the variation of $r_{1}$ and $r_{2}$ is the same with $r_{\max }=4 \cdot 10^{5} \mathrm{~W}$, while the maximum value for $r_{3}$ is $22 \cdot 10^{5} \mathrm{~W}$ and $N_{r}$ is also equals to 100. Expression (15), requires the load and generation profiles during a year. For this particular system, 24 load curves $\breve{\mathcal{T}}^{h}(t)$ were generated using the software HOMER described in [27]. The time parameter $t$ is varying from 1 to $8760 h$ with a time step of $1 h$.

A very accurate solution can be represented with only 18 PGD modes in 6 iterations as can be seen in the convergence diagram of the nonlinear algorithm Fig. 6 . In this case the tolerances are $\epsilon_{f p}=10^{-8}, \epsilon_{g}=10^{-7}$ and $\epsilon_{n}=10^{-5}$. If $N_{t}$ is the number of hours in a year, the whole parametric space is represented by a considerable amount of information $N_{q} \cdot N_{T} \cdot N_{d} \cdot N_{t} \approx 1.14 \cdot 10^{10}$, however the same information

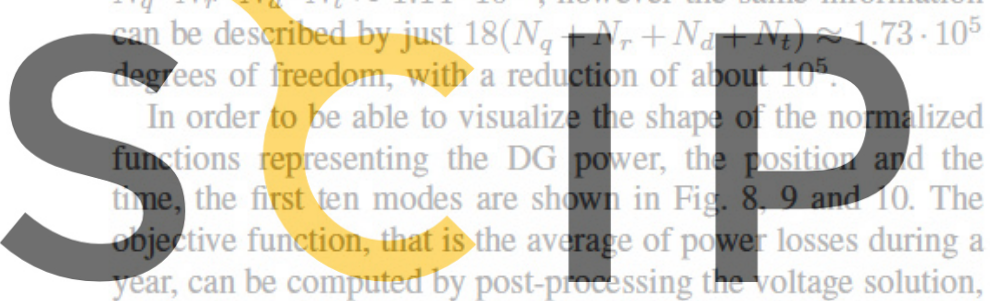
see Fig. 7. The optimal position of the DGs are la724, $l b 726$

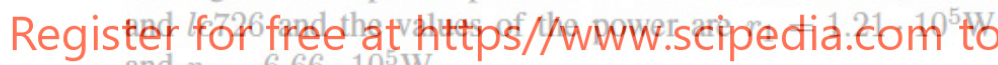
and $r_{2}=6.66 \cdot 10^{5} \mathrm{~W}$

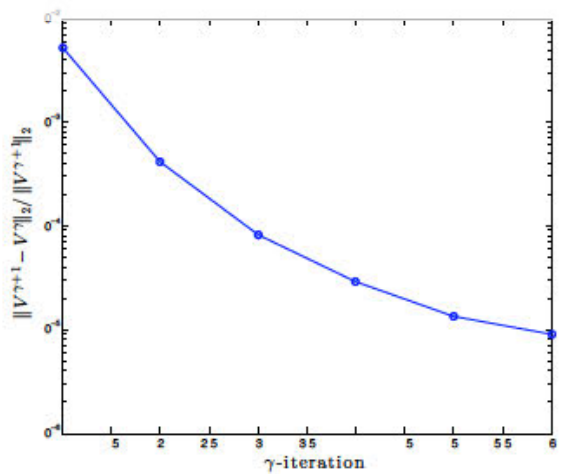

Fig. 6: Nonlinear PGD convergence diagram

\section{Conclusions}

This work presented the results obtained when applying a non-linear solver combined with the Proper Generalized Decomposition to the Parametric Power Flow problem for optimal location and sizing of distributed generation.

The "offline-online" approach shown allows to compute once and for all the solution in the "offline" phase while

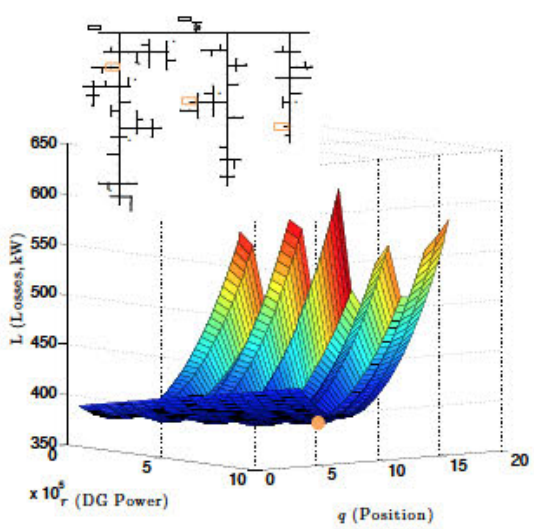

Fig. 7: Reconstructed System Losses

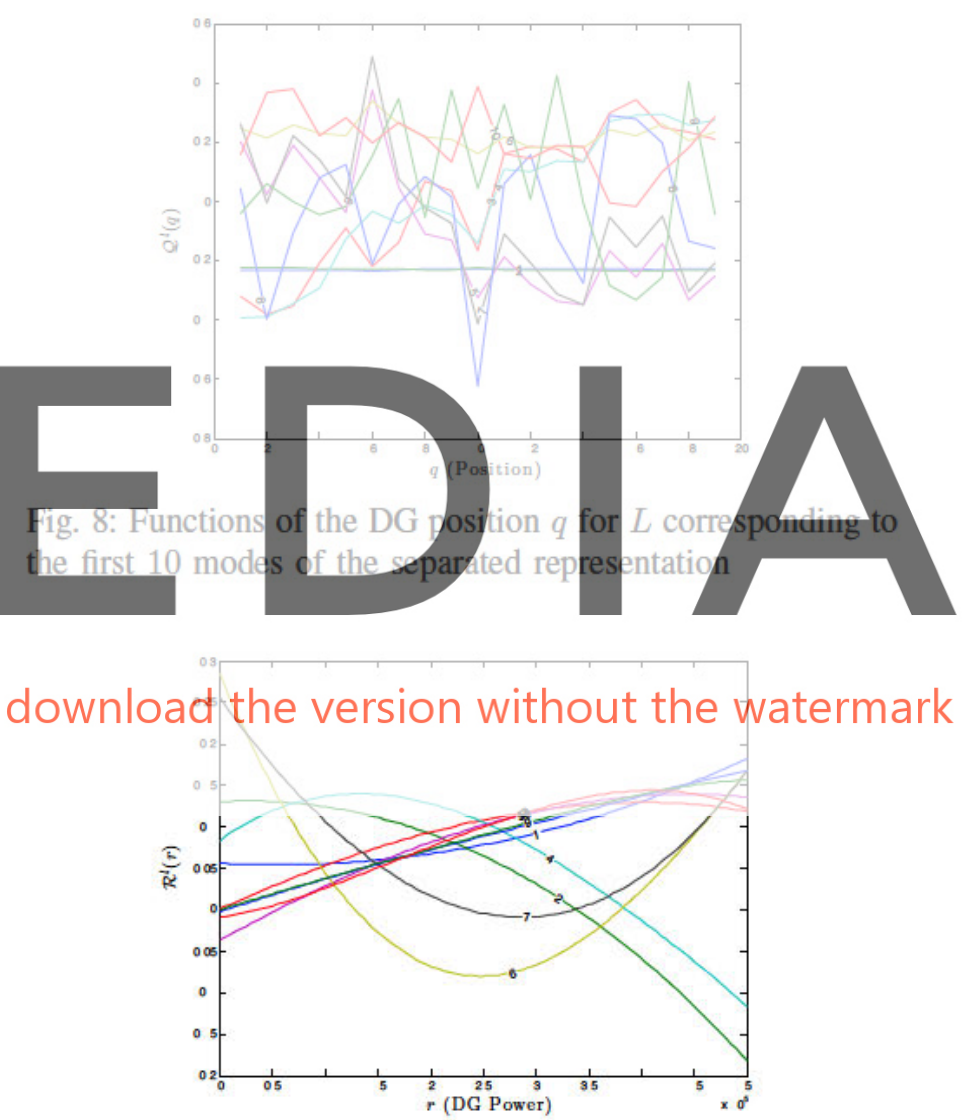

Fig. 9: Functions of the DG output active power $r$ for $L$ corresponding to the first 10 modes of the separated representation

optimization can be performed effortlessly in the "online" step, because of the objective function is explicitly available. The PGD technique allows to store the information associated to the resolution of a highly dimensional problem in a compressed separated variables format. Furthermore, the algorithm to compute solution itself scales linearly with the dimensionality of the problem, since each mode of the separated representation is computed individually.

From the obtained results, it is possible to see how the 


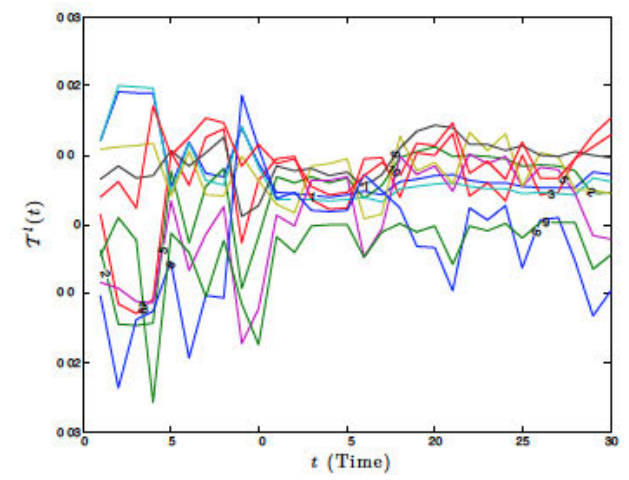

Fig. 10: Functions of the time $t$ for $L$ corresponding to the first 10 modes of the separated representation

reduction rate becomes evident as the dimensionality of the problem increases. In the eight dimensional problem, the objective function is computed in the whole parametric space with a reduction rate of about $1.73 \cdot 10^{5}$.

\section{ACKNOWLEDGMENTS}

The authors of this work would like to thank prof. Juan Antonio Martinez Velasco and Luis Gerardo Guerra Sanchez from the
Politéc
previding

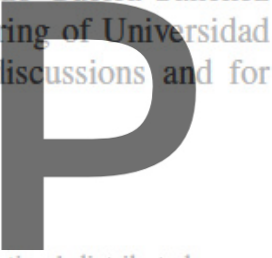

1] Georgilakis, P. S. and Hatziargyriou, N. D., "Optimal distributed generation placement in power distribution networks: models, methods, and

Register for freed, at https/fwww. Scipedia.com, to

[2] S. D. M. Shareef and T. V. Kumar, "A review on models and methods for optimal placement of distributed generation in power distribution system," in internationai Journai of Education and Appiied Research 2014, vol. 4, pp. 161-169.

[3] Nara, K. and Hayashi, Y. and Ikeda, K. and Ashizawa, T., "Application of tabu search to optimal placement of distributed generators," in IEEE Power Engineering Society Winter Meeting. Conference Proceedings, 2001, C, pp. 918-923.

[4] Mithulananthan, N. and Oo, Than. and Phu, Le Van., "Distributed generator in power distribution placement system using genetic algorithm to reduce losses," in Thammasat International Journal of Science and Technology, vol. 9, 2004.

[5] Georgilakis, Pavlos S and Hatziargyriou, Nikos D, "Analytical approaches for optimal placement of distributed generation sources in power systems," in IEEE Transactions on Power Systems, vol. 19, no. 4, pp. 2068-2076, 2004.

[6] Acharya, Naresh and Mahat, Pukar and Mithulananthan, N., "An analytical approach for DG allocation in primary distribution network," in International Journal of Electrical Power \& Energy Systems, vol. 28, no. 10, pp. 669-678, 2006.

[7] Martinez, J.A. and Guerra, Gerardo, "Optimum placement of distributed generation in three-phase distribution systems with time varying load using a Monte Carlo approach," in Power and Energy Society General Meeting, 2012 IEEE, pp. 1-7.

[8] Atwa, Y.M. and El-Saadany, E.F., "Probabilistic approach for optimal allocation of wind-based distributed generation in distribution systems," in IET Renewable Power Generation, vol. 5, no. 1, pp. 79-88, 2011.

[9] Griffin, T. and Tomsovic, K. and Law, A., "Placement of dispersed generation systems for reduced losses," in Proceedings of the 33rd Hawaii International Conference on System Sciences-2000, pp. 1-9.
[10] Lin, G. and Elizondo, M. and Lu, S. and Wan, X., "Uncertainty quantification simulations in dynamic simulations of large-scale power system models using the high-order probabilistic collocation method on sparse grids," in Int. J. Uncert. Quant. 2014, vol. 4, no. 3, pp. 185-204.

[11] Zhang, Z. and Nguyen, H. D. and Turitsyn, K. and Daniel, L., "Probabilistic power flow computation via low-rank and sparse tensor recovery," in review, presented in IEEE Transactions on Power System, 2015.

[12] Zhang, H. and Li, P., "Application of sparse-grid technique to chance constrained optimal power flow," in Generation, Transmission and Distribution, IET 2013, vol. 7, no. 5, pp. 491-499.

[13] Tang, J. and Ni, F. and Ponci, F. and Monti, A., "Dimension-adaptive sparse grid interpolation for uncertainty quantification in modern power systems: probabilistic power flow," in IEEE Transactions on Power System 2015, vol. PP, no. 99, pp. 1-13.

[14] Hinze, M. and M. Kunkel, "Discrete empirical interpolation in POD model order reduction of drift-diffusion equations in electrical networks," Scientific Computing in Electrical Engineering SCEE 2010, Mathematics in Industry, pp. 423-431. Springer Berlin Heidelberg.

[15] Parrilo, P., S. Lall, and F. Paganini, "Model reduction for analysis of cascading failures in power systems," in Proceedings of the 1990 American Control Conference, vol. 6, pp. 4208-4212. 2013

[16] Chinesta, F. and Ladevèze, P. and Cueto, E., "A short review on model order reduction based on proper generalized decomposition," in Archives of Computational Methods in Engineering, v.18, no. 4, pp. 395-204, 2011.

[17] Chinesta, F. and Ammar, A, and Cueto, E. "Recent advances and new challenges in the use of the proper generalized decomposition for solving multidimensional models," in Archives of Computational methods in Engineering, v.17, no.4, pp. 327-350, 2010.

[18] F. Chinesta, A. Leygue, F. Bordeu, J. Aguado, E. Cueto, D. Gonzalez, I. Alfaro, A. Ammar, and A. Huerta, "PDG-based computational vade-

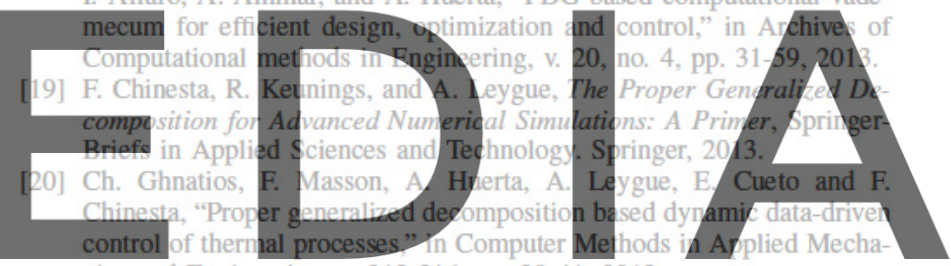
nics and Engineering, v. 213-216, pp. 29-41, 2012.

[21] M.S. Aghighi, A. Ammar, C. Metivier, M. Normandin, and F. Chinesta.

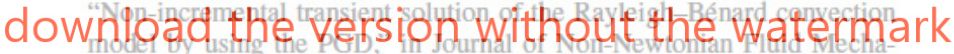
nics, v. 200, pp. 65-78, 2013.

[22] S. Niroomandi, I. Alfaro, D. González, E. Cueto, and F. Chinesta, "Modei order reduction in hypereiasticity: a proper generaized decomposition approach," in International Journal for Numerical Methods in Engineering, v. 96, no. 3, pp. 129-149, 2013.

[23] T.-H. Chen, M.-S. Chen, K.-J. Hwang, P. Kotas, and E. Chebli, "Distribution system power flow analysis-a rigid approach," in IEEE Trans. Power Del., vol. 6, no. 3, pp. 1146-1152, Jul 1991.

[24] D. Borzacchiello, M.H. Malik, F. Chinesta, R. García-Blanco, and P. Diez, "Unified formulation of a family of iterative solvers for power systems analysis," in review, presented in IEEE Electric Power Systems Research, 2016.

[25] D. Borzacchiello, F. Chinesta, R. García-Blanco, and P. Diez, "Introduction to the Proper generalized decomposition for the solution of the parametrized power equations," in review, presented in IEEE Transactions on Power System, 2016.

[26] G. Guerra and J.A. Martinez-Velasco, "Optimum allocation of distributed generation in multi-feeder systems using long term evaluation and assuming voltage-dependent loads," Sustainable Energy, Grids and Networks, in press.

[27] HOMER ENERGY LLC (2015). HOMER PRO [Computer software]. Colorado, USA. Retrieved from http://www.homerenergy.com/ HOMER_pro.html 